\title{
Various driving mechanisms for generating motion of colloidal particles
}

\author{
A Erbe ${ }^{1}$, M Zientara ${ }^{2}$, L Baraban ${ }^{2}$, C Kreidler ${ }^{2}$ and P Leiderer ${ }^{2}$ \\ ${ }^{1}$ Universität Konstanz, FB Physik und Zukunftskolleg, Universitätsstr. 10, D-78457 Konstanz, \\ Germany \\ ${ }^{2}$ Universität Konstanz, FB Physik, Universitätsstr. 10, D-78457 Konstanz, Germany \\ E-mail: Artur.Erbe@uni-konstanz.de
}

Received 27 April 2008, in final form 10 July 2008

Published 10 September 2008

Online at stacks.iop.org/JPhysCM/20/404215

\begin{abstract}
Driven colloidal particles exhibit a number of interesting nonequilibrium effects. In order to study these effects the driving mechanisms have to be understood in detail. In this paper we

- show three different driving mechanisms: driving by gravity, by a gradient in a magnetic field, and by a local chemical reaction. The first mechanism can be used to drive a large variety of particles, while the latter two require specially designed particles. We will describe the design of such particles and discuss differences and similarities of the driving mechanisms.
\end{abstract}

\section{Introduction}

Colloidal particles are excellent model systems for many interparticle interactions. They have been used to study phase behavior of hard spheres [1] and charged colloidal particles [2] in three dimensions, as well as for studies of the two-stage melting process of particles in two-dimensional configurations [3]. Particles, which are confined on lattice sites, show patterns which result from the competition of the interparticle energies with the energies from the interaction of the particles with the potential defining the lattice [4]. Particles with predefined static magnetic dipole moments can be used as models for spin interactions of atoms on various lattice geometries [5]. The influence of spatial boundaries leads to a competition between the interparticle interactions and the conditions imposed by the external confining potential [6-8]. If such an ensemble of particles is driven into motion, interesting and unexpected reconfigurations of the particle arrangements take place due to the collective motion of the particles [9]. Other effects are, for example, hydrodynamic effects due to the presence of a surrounding solvent [10] and lane formation, if two kinds of particles are driven in opposite directions [11].

It is therefore worthwhile to study the effects of various driving mechanisms on single colloidal particles and ensembles of such particles. In this work we want to present three driving mechanisms acting on two different kinds of colloidal particles: on the one hand, gravitational driving and driving by means of a gradient in a magnetic field, both on superparamagnetic particles, and, on the other hand, driving by a chemical reaction at the surface of the particles on capped colloids. The particles are described in section 2 and the driving methods in section 3 .

\section{Particles}

Superparamagnetic particles (commercially available from Dynal) are created by $\mathrm{Fe}_{3} \mathrm{O}_{4}$ inclusions in a polystyrene matrix. The size of the inclusions is of the order of several nanometers; thus the magnetic moments of the inclusions can follow directly an externally applied magnetic field leading to the superparamagnetic behavior of the whole particle. The diameter of our particles is about $4.55 \mu \mathrm{m}$. Interactions between such superparamagnetic particles are long-ranged. In the case of a two-dimensional arrangement of the particles, which are polarized perpendicular to the plane of the particles, the potential of the magnetic dipoles is given by

$$
V(r)=\frac{\mu_{0}}{4 \pi} \frac{M^{2}}{r^{3}}
$$

Here $r$ is the distance between particles. In the case of the superparamagnetic particles $M$ is

$$
M=M_{0}\left(\operatorname{coth}(\alpha B)-\frac{1}{\alpha B}\right),
$$

with $M_{0}=6 \times 10^{-13} \mathrm{Am}^{2}$ and $\alpha=390 \mathrm{~T}^{-1}$.

For some applications, such as the generation of an autonomous motion, it is desirable that the particles have 
asymmetric properties. Such asymmetry can be achieved by the evaporation of a metallic cap onto silica beads. In our experiments the diameter of the particles is $4.82 \mu \mathrm{m}$. A metal (palladium in the cașe of the present study) is evaporated on one side of the particles. This can be realized if the particles are first deposited on a substrate in a monolayer, either by evaporation of the solvent from a droplet [12] or by spin coating. The metal is deposited on top of such a monolayer in a subsequent step by thermal evaporation. The particles can then be suspended from the substrate by dipping the substrate in water or by sonicating the samples in water. The resulting suspension is enriched by centrifuging.

\section{Driving mechanisms}

\subsection{Gravitational driving}

The mechanism of gravitational driving has been demonstrated by Köppl et al [9]. The suspension of superparamagnetic particles is put on to a quartz substrate, which is coated by a resist (Microchem SU-8) in order to prevent sticking of the particles to the substrate. A second quartz substrate is put on top of this, separated from the bottom by a rubber $\mathrm{O}$ ring. The whole cell is sealed by a UV glue (Norland Optical Adhesive 60 ) to prevent unwanted evaporation of the solvent. The dimensions of the cell are of the order of a few millimeters. We therefore consider the system as an unbound system on the scale of typical particle sizes $(4.5 \mu \mathrm{m})$.

For gravitational driving the sample cell is tilted by a welldefined angle $\theta$. The strength of the driving force is then given by

$$
F_{\text {grav }}=m_{\text {rel }} g \sin \theta,
$$

$m_{\text {rel }}$ being the reduced mass of the particle $m_{\text {rel }}=\left(\rho_{\text {par }}-\right.$ $\left.\rho_{\text {sol }}\right) V_{\text {par }}$, where $\rho_{\mathrm{par}}$ and $\rho_{\text {sol }}$ are the densities of the particle and the solvent, respectively, and $V_{\mathrm{par}}$ is the volume of the particle. The tilt can be generated by either tilting the whole set-up or tilting the sample with respect to the set-up. The latter has the advantage that the optical set-up can be built more reliably and is therefore used in the current experiments. It should be mentioned that in a closed cell the particles move with respect to the solvent. Therefore hydrodynamic interactions can play an important role in this set-up.

\subsection{Driving by a gradient in the magnetic field}

The particles possess a magnetic moment, which depends on the value of an external magnetic field (the polarizing field $B_{\text {pol }}$ in relation (2)). If a second magnetic field, $B_{\text {driv }}$, is superimposed the interaction between the magnetic dipole and the driving field leads to a force proportional to the gradient $\vec{\nabla} \vec{B}_{\text {driv }}$. In our set-up we use two pairs of magnetic coils. One generates a homogeneous field directed perpendicular to the sample's substrate $\left(B_{\text {pol }}\right)$, the other pair generates a field, which is oriented in the plane of the particles' motion, $B_{\text {driv. }}$. This field is generated by driving the current through the two coils not in parallel, but in opposite directions. The sizes and the distance between the coils were chosen so that $B_{\text {driv }}$ is linear in the area of the motion of the particles.
Because the magnetic moments of the superparamagnetic particles are induced by the applied magnetic field, the gradient in the driving field leads to a change in the magnetic moment of the particles. The simple relation $F \propto \vec{\nabla} \vec{B}$ is then no longer correct and has to be replaced by

$$
F_{\text {mag }}=\vec{\nabla} \vec{B}_{\text {driv }} \frac{\mathrm{d}\left(x M_{x}\right)}{\mathrm{d} x},
$$

where $x$ is the distance from the point where $B_{\text {driv }}=0$ and $M_{x}$ is the component of the magnetic moment $\vec{M}$ in the direction of the driving field. For typical conditions in our experiments the value of the force is of the order of $10^{-14} \mathrm{~N}$. $M$ can be estimated by the first nonzero term of its Maclaurin's series for $B: M=M_{1} B$, where $M_{1}=3^{-1} \alpha M_{0}$. (This is a good estimate for fields $<2 \mathrm{mT}$.) Thus we can write $F_{\text {mag }}$ in a simpler form:

$$
F_{\text {mag }}=2 M_{1}\left(\vec{\nabla} \vec{B}_{\text {driv }}\right)^{2} x
$$

which shows the direct relation between $F_{\text {mag }}$, the gradient and the distance from the center of the driving field $x$. It is confirmed by measurements of velocities of single particles as a function of $x$ and as a function of the gradient.

\subsection{Catalytic reaction at the cap}

It is well known that $\mathrm{H}_{2} \mathrm{O}_{2}$ undergoes a catalytic decomposition at the surfaces of transition metals. The capped colloids, which were presented in section 2 , are metallic on one hemisphere only. Therefore the decomposition of hydrogen peroxide is much stronger on the palladium-coated side of the sphere than on the bare $\mathrm{SiO}_{2}$. The Pd-catalyzed decomposition of hydrogen peroxide in water solution can be written as $2 \mathrm{H}_{2} \mathrm{O}_{2} \rightarrow 2 \mathrm{H}_{2} \mathrm{O}+\mathrm{O}_{2}$. The balance equation shows that the ratio of reactants to products is $2: 3$. Consequently a local gradient of the chemical potential around the particle is formed. The catalytic decomposition of the hydrogen peroxide at the palladium surface of the particle leads to an increase of the number of molecules there, resulting in the appearance of a difference in osmotic pressure. This imposes propulsion of the particle from the region of high pressure (palladium side) into a region of low pressure (uncoated side) towards the silica part. We can estimate the heating of the whole system from the energy of the hydrogen peroxide decomposition $\left(98 \mathrm{~kJ} \mathrm{~mol}^{-1}\right.$ ). Under the assumption that even in rather dense suspensions less than $1 \%$ of hydrogen peroxide will be decomposed, we derive a temperature change of $1 \mathrm{~K}$ for a typical volume of $200 \mu \mathrm{l}$. Therefore heating effects are unlikely to influence the driven motion of the particles.

This chemical reaction has been suggested for the generation of motion of objects catalyzed by platinum [13] and described by Howse et al [14] for the propulsion of the platinum-coated polystyrene beads, where platinum was used as the catalyst. A similar driving mechanism was used to move bi-composite metallic rods [13] and platinum-coated plates [15]. Because the motion in this case is determined by the ability to produce the chemical gradient locally, the geometry and shape of the object can play an important role in the generation of its motion. We can therefore expect that changes 
a)

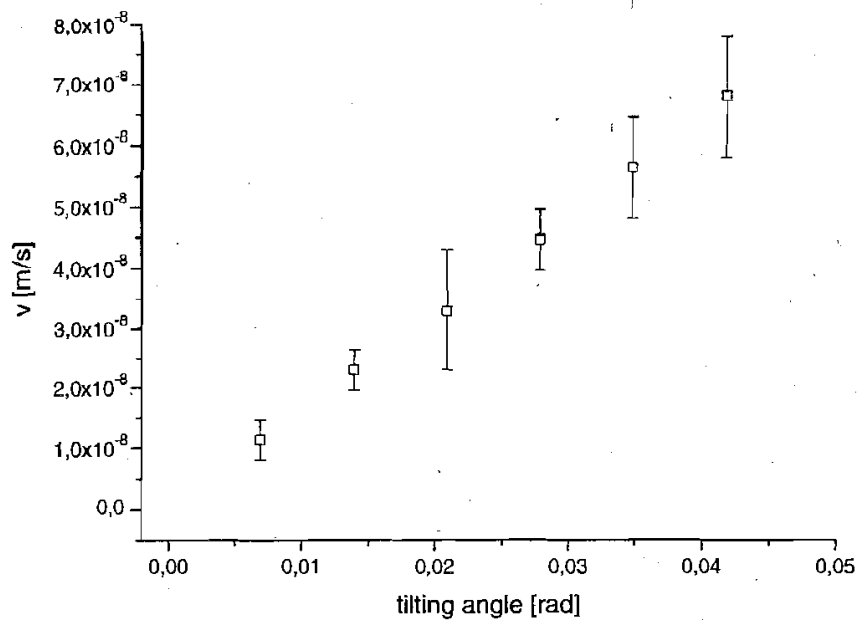

b)

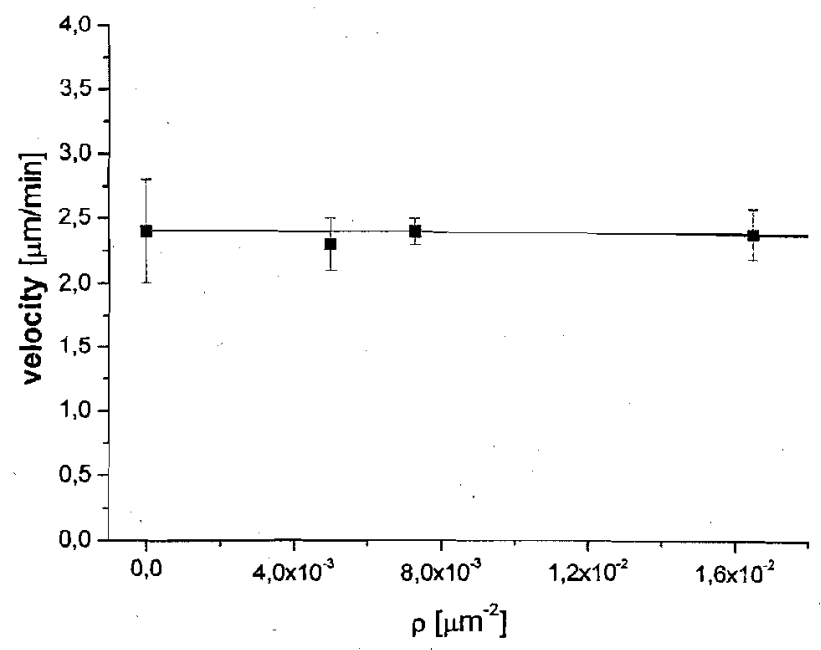

Figure 1. (a) Dependence of particle velocity on the tilting angle $\theta$. (b) Dependence on changes in particle density.

in the shape of the capped colloids to non-spherical particles can lead to a preferred direction in the driven motion of these particles.

A suspension of capped colloids was prepared by mixing the particles with hydrogen peroxide solutions of different concentrations, prepared by diluting a $30 \%$ hydrogen peroxide solution with water. The suspension was placed between two glass plates, separated by a glass spacer. The motion of the colloidal particles was directly imaged by means of video microscopy.

\section{- 4. Experimental results}

All experiments are conducted in a video microscopy setup. Before the first measurements of particle velocities the set-up is carefully oriented horizontally in order to remove any drift arising from an unintentional residual tilting of the set-up. It has to be mentioned that, due to hydrodynamic interactions, interparticle interactions are expected when the particles are measured moving as ensembles. Furthermore the magnetic moments of the superparamagnetic particles interact on long length scales. We therefore performed measurements comparing the behavior of ensembles of particles with the behavior of individual particles. This procedure was not necessary for the case of catalytically driven particles, because there every particle is driven individually by the reaction with the fuel molecules.

Figure 1(a) shows the dependence of the velocity on various tilt angles $\theta$. The expected linear dependence for small values of $\theta$ is observed. Without hydrodynamic interactions the velocity of a particle with radius $R$ is expected to be given by equating the force ( 3 ) with the friction given by Stokes' law:

$$
F=6 \pi \eta R v
$$

From this estimate we expect a velocity of $v=0.21 \mu \mathrm{m} \mathrm{s}^{-1}$ for a particle driven at a tilt angle of $\theta=2^{\circ}$ (with the viscosity of water $\eta=1 \times 10^{-3} \mathrm{~Pa}$ s and the particle density $\rho=1.5 \times 10^{3} \mathrm{~kg} \mathrm{~m}^{-3}$ ). This deviates strongly from the experimentally measured value $\left(v=0.05 \mu \mathrm{m} \mathrm{s}^{-1}\right)$. This indicates that the proximity of the surface causes friction, which slows down the motion of the particles. In order to check whether effects of hydrodynamic interactions arise, when an ensemble of particles is driven gravitationally, we repeated the same measurement at various particle densities. Figure 1(b) shows that we could not observe a change in velocity due to changes in density at $\theta=2^{\circ}$. We therefore conclude that hydrodynamic interactions between the particles are weak in our system at these moderate densities and particle velocities.

The velocity of the particles driven by a gradient in the magnetic field depend, according to equation (5), linearly on the position $x$ and quadratically on the gradient in the magnetic field $\vec{\nabla} \vec{B}$. Measurements shown in figure 2 confirm both relations. This shows that the particles are driven by a welldefined gradient of the magnetic field in the experiment. In order to test the magnitude of the magnetic force $F_{\text {mag }}$ the force was compared to gravitational forces. For this experiment we tilted the sample in the direction which causes a force opposing the magnetic drive. Thus the particles are pulled 'uphill' by the magnetic force as long as $F_{\text {mag }}>F_{\text {grav }}$ and flow 'downhill' as soon as $F_{\text {mag }}<F_{\text {grav }}$. When plotting the absolute value of the velocities of the particles $|v|$ versus the tilting, angle $\theta$ at a certain value of the gradient in a magnetic field $\left(0.386 \mathrm{~T} \mathrm{~m}^{-1}\right)$ we therefore obtain a minimum at $F_{\text {mag }}=F_{\text {grav }}$, as shown in figure 3(a). This measurement leads to a value of $1 \times 10^{-14} \mathrm{~N}$ for equilibrium. When calculating the force for the given field gradient from equation (5) we observe a value of $2.3 \times 10^{-14} \mathrm{~N}$. To further characterize the driving mechanism in the magnetic field we measure the velocities of the particles at different values of the force. Figure 3(b) depicts this dependence. The force values' are calculated according to equation (5). Both methods show that the measured values of the magnetic force deviate strongly from the values which 

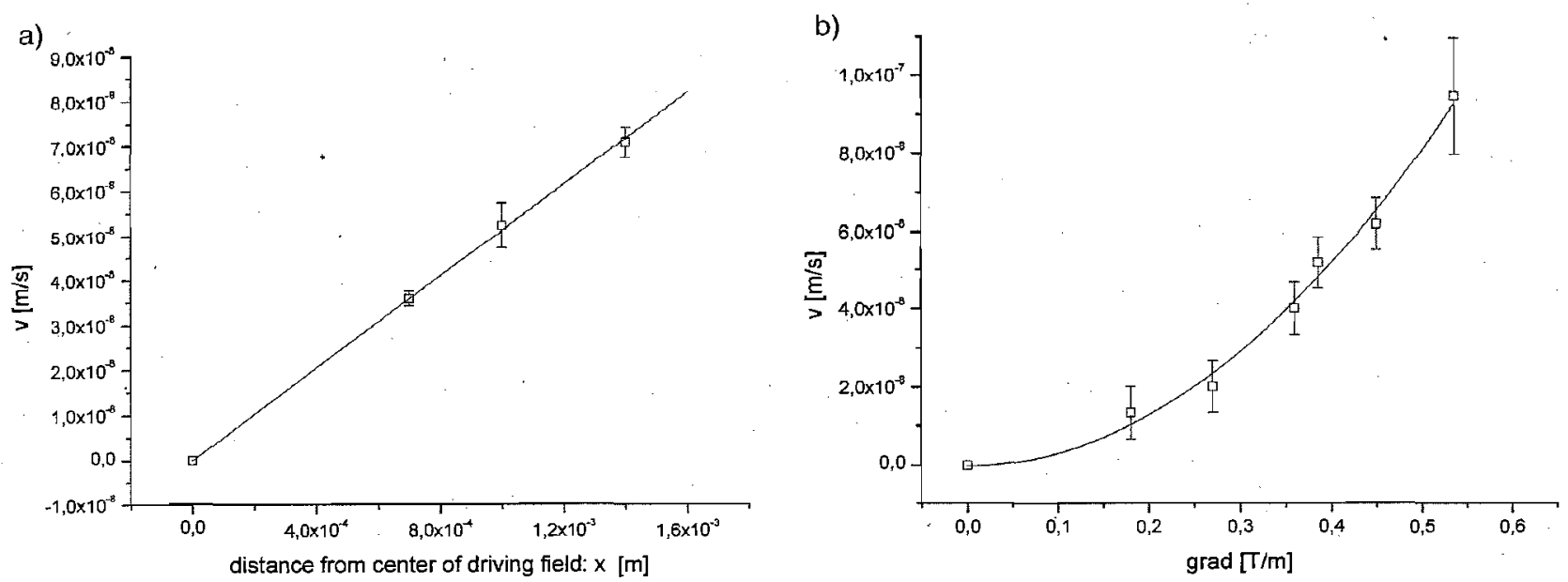

Figure 2. (a) Dependence of particle velocity on the position in the gradient of the magnetic field $x$. (b) Dependence on the strength of the gradient $\vec{\nabla} \vec{B}$. The full lines represent dependences $v \propto x$ and $v \propto(\vec{\nabla} \vec{B})^{2}$, respectively.

a)

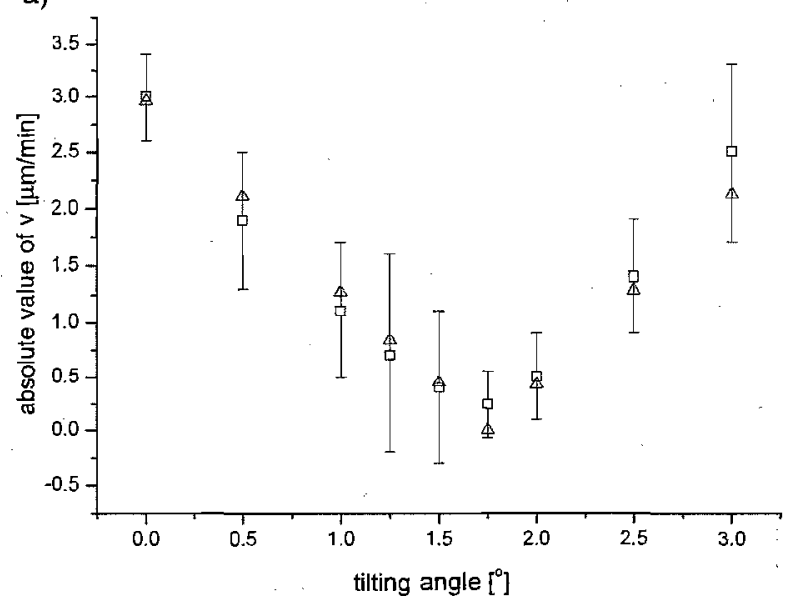

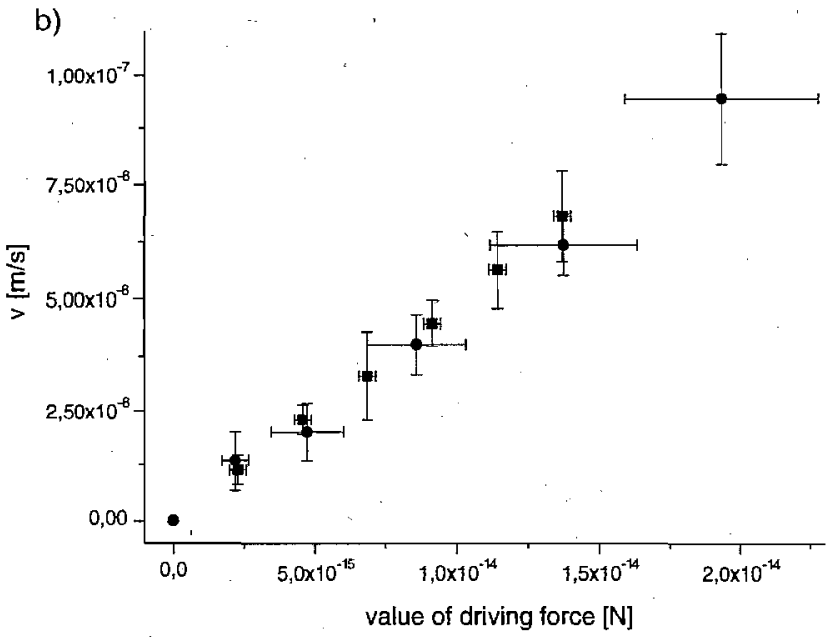

Figure 3. (a) Dependence of the velocities of the particles on the tilting angle $\theta$ at a gradient in the magnetic field of $0.386 \mathrm{~T} \mathrm{~m}^{-1}$. The distance from the center of the magnetic field is $x=1 \mathrm{~mm}$. (b) Comparison between the velocities of the particle in magnetic and gravitational driving for forces calculated according to equations (2) (squares) and the corrected equation (7) (circles).

can be calculated from equation (5). We estimated the errors coming from our determination of the velocities (shown is the spread of several measurements) and the determination of the gradient of the magnetic field, which was measured using a commercial Hall probe. The result, which is shown in figure 3(b), indicates that the discrepancy is beyond the experimental error. Differences in friction or hydrodynamic interactions in the two driving mechanisms cannot explain this result, because the position of the minimum in figure 3(a) is not affected by such influences. One possible reason for these differences is the fact that the polarization of the superparamagnetic particles, which is induced by the motion from a region of lower $B_{\text {driv }}$ to a region of larger $B_{\text {driv }}$, costs energy. This loss in energy leads to a 'friction' term in the force $F_{\text {mag }}$, which is again proportional to $(\vec{\nabla} \vec{B})^{2}$. Thus the total force can be rewritten as

$$
F_{\text {mag }}=(2-\gamma) M_{1}\left(\vec{\nabla} \vec{B}_{\text {driv }}\right)^{2} x
$$

with a parameter $\gamma$, which has to be calculated from the experimental comparison between magnetic and gravitational driving. For our measurements we find a value of $\gamma \approx 1$.

Unlike the two previous cases the catalytically driven particles perform a motion, which is induced by their own chemical gradient. Driving force always points in the direction of the particles' cap, therefore the direction of the motion is defined by the particles' axis. Although such a particle moves directly at short timescales, its motion is found to be random at large timescales because of processes of rotational diffusion, which change the direction of the cap and the driving force. This motion can then be described by an effective diffusion constant [14]:

$$
D_{\text {eff }}=D_{0}+\frac{1}{4} v^{2} \tau_{\mathrm{R}}
$$

with $D_{0}$ the diffusion constant without addition of $\mathrm{H}_{2} \mathrm{O}_{2}$ and $\tau_{\mathrm{R}}$ the rotational diffusion constant. This constant depends on the content of $\mathrm{H}_{2} \mathrm{O}_{2}$ in the solution. We determined it for 


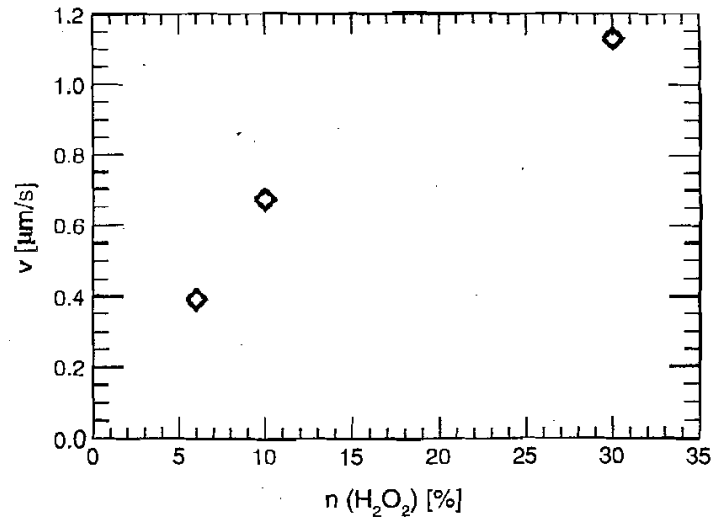

Figure 4. Dependence of the velocity of capped particles in various concentrations of $\mathrm{H}_{2} \mathrm{O}_{2}$.

various concentrations of $\mathrm{H}_{2} \mathrm{O}_{2}$ and could derive velocities of the capped particles in these concentrations (figure 4). The velocity of the particles depends linearly on the concentration of $\mathrm{H}_{2} \mathrm{O}_{2}$ in the solution up to $20 \% \mathrm{H}_{2} \mathrm{O}_{2}$. It is found to be about $1 \mu \mathrm{m} \mathrm{s}^{-1}$ at $20 \% \mathrm{H}_{2} \mathrm{O}_{2}$. At larger contents of $\mathrm{H}_{2} \mathrm{O}_{2}$ the velocities start to saturate because the reaction educts cannot be provided at sufficient rates to the particles' surface any more. If we assume that hydrodynamic interactions can be neglected in this case as well, we can estimate a maximum driving force of $0.25 \mathrm{pN}$ for a single capped particle.

Catalytic motion of colloidal particles can find many applications, for instance, transportation of synthetic or biological objects. For this purpose the possibility to control the direction of motion becomes.important. In order to achieve directed motion of such particles, the processes of rotational diffusion, which lead to random changes of the orientation of the metallic caps in the solvent, must be excluded. This can be realized by modifying the particles' caps by deposition of additional ferromagnetic layers, which will generate a permanent magnetic moment for every colloidal particle [5]. The motion of such modified colloids in a solution of $\mathrm{H}_{2} \mathrm{O}_{2}$ can be directed by applying a homogeneous external magnetic field, which will align the particles' caps along field lines [16]. The velocities for such a motion are not affected by weak magnetic fields and are therefore comparable to velocities shown in figure 4.

\section{Conclusion}

To summarize we investigated three different driving mechanisms for colloidal particles. The forces which act on the particles are comparable in all cases and are on the pN scale. The comparable magnitude of the driving forces will allow us to investigate multi-component ensembles of particles driven in different directions. One possible phenomenon, which can be observed in such systems, is the formation of lanes of particles, which move together in the same direction [11].

\section{Acknowledgments}

This work was supported by the DFG via the SFB TR6 and the IRTG on soft condensed matter.

\section{References}

[1] Gasser U, Weeks E R, Schofield A, Pusey P N and Weitz D A 2001 Science 292258

[2] Leunissen M, Christova C, Hynninen A-P, Royall C, Campbell A, Imhof A, Dijkstra M, Roij R v and Blaaderen A v 2005 Nature 437235

[3] Zahn K, Lenke R and Maret G 1999 Phys. Rev. Lett. 822721

[4] Mangold K, Leiderer P and Bechinger C 2003 Phys. Rev. Lett. 90158302

[5] Baraban L, Makarov D, Albrecht M, Rivier N, Leiderer P and Erbe A 2008 Phys. Rev. E 77031407

[6] Haghgooie R and Doyle P 2005 Phys. Rev. E 75061406

[7] Haghgooie R and Doyle P S 2007 Phys. Rev. E 75061406

[8] Bubeck R, Bechinger C, Neser S and Leiderer P 1999 Phys. Rev. Lett. 823364

[9] Koppl M, Henseler P, Erbe A, Nielaba P and Leiderer P 2006 Phys. Rev. Lett. 97208302

[10] Lutz P L C, Kollmann M and Bechinger C 2004 J. Phys.: Condens. Matter $16 \mathrm{~S} 4075$

[11] Dzubiella J, Hoffmann G and Lowen H 2002 Phys. Rev. E 650214021

[12] Burmeister F, Schafle C, Matthes T, Bohmisch M, Boneberg J and Leiderer P 1997 Langmuir 132983

[13] Robbins M H and Drago R S 1997 J. Catal. 170295

[14] Howse J R, Jones. R A L, Ryan A J, Gough T, Vafabakhsh R and Golestanian R 2007 Phys. Rev. Lett. 99048102

[15] Ismagilov R, Schwartz A, Bowden N and Whitesides G 2002 Angew. Chem. 114674

[16] Baraban L, Kreidler C, Makarov D, Leiderer P and Erbe A 2008 Preprint 0807.1619v1. [cond-mat] 